\title{
The Outcome of Darning Method of Inguinal Hernia Repair Using Polypropylene in Sir Ganga Ram Hospital, Lahore
}

\author{
A A NAAZAR MS ARSHAD A WAHEED MS. QURESHI \\ Department of Surgery, Sir Ganga Ram Hospital, Lahore \\ Correspondence to Dr. Ali A Naazar E. mail: aliabidnaazar@msn.com.
}

\begin{abstract}
Objectives: To valuate the darning method of inguinal hernia repair with polypropylene. Material and Methods: 300 patients of unilateral inguinal hernia were admitted. After thorough investigations, all patients were operated for hernia repair by standard procedure using No.1 polypropylene. Patients were operated under, spinal or general anesthesia. Patients were followed up at one week, six weeks and six months after operation for wound healing and complications. Results: Out of 300 patients, ranging in age from 20 to 70 years, 250 patients were given spinal anaesthesia, 45 patients $(20 \%)$ were operated under local anesthesia. 196 patients were discharged from the hospital on the following day. 3 patients went into urinary retention and one patient had large haemotoma requiring exploration. Recurrence rate was $1 \%(n=3)$. Conclusion: Darning method of inguinal repair with polypropylene is a safe and cheaper method of hernia repair.

Key word: Inguinal hernia, Polypropylene Darn.
\end{abstract}

Hernia by definition is the protruding of an organ or part of an organ through the wall that normally contains it. Hernia occupies $10-15 \%$ of all surgical procedures, $80 \%$ are inguinal and $92 \%$ are in men. $18 \%$ of this occurs below 15 yrs of age, $26 \%$ between 15 and 44 yrs, $30 \%$ between 45 and 64 yrs and $26 \%$ above 65 yrs indirect inguinal hernia occurs in $55 \%$ and direct in $30 \%$ while pantaloon hernia in $15 \%$ cases ${ }^{\prime}$. There is a growing body of evidence that adult male inguinal hernias are associated with impaired collagen metabolism and weakening of the fibro connective tissue of the groin, current physical activity is only considered a secondary or triggering factor in the development of inguinal hernias ${ }^{2,3}$. There are many ways of repairing an inguinal hernia, with over 80 operative techniques described since 1887 when Bassini reported his method. Extensive clinical research has been undertaken to assess outcome following inguinal hernia repair ${ }^{4}$.

High recurrence rates using fascia for inguinal hernia repair or suturing under tension prompted the development of minimal tension nylon darn, and then polypropylene mesh, to reinforce the posterior wall of the inguinal canal during inguinal hernia repair. A nylon darn, originally described by Maloney et al, is a cheap and effective way of repairing a hernia ${ }^{5}$. The recurrence rate reported from the original series was $0.8 \%{ }^{6}$. More recently the use of polypropylene mesh has become popular because of excellent results reported by Lichtenstein et $\mathrm{al}^{7}$. There are laparoscopic approaches of hernia repair, which so for has not gained much popularity as only $5 \%$ of hernia repair are done this way.

The aim of this study was to evaluate the polypropylene darning method in the treatment of primary inguinal hernia in men, assessing complications, postoperative pain, return to normal activities and early recurrence.

Material and methods:

A total 300 cases of hernia were included in the study done over a period of 36 months from July 2002 to June 2005 .
Age range was between 24 and 70 yrs. All patients were evaluated by taking careful history taking, physical examination. Hemoglobin, blood Urea. Blood Sugar testing and where appropriate ECG and chest radiograph. $65 \%$ of patients were secondary referrals. Only unilateral hernias were included. Bilateral, irreducible, obstructed, strangulated and recurrent hernias were excluded. 250 cases underwent general anesthesia while the cases on spinal and local anesthesia were 50 each. They were all submitted to the same standard procedure using No. 1 polypropylene which is monofilament, non absorbable, relatively biologically inert and do not adhere to tissues. The first stitch was taken from the pubic tubercle and a darn was made locking each stitch between tendinous conjoint tendon and inguinal ligament, without tension in an interwoven fashion. The procedure was tension free. In patients of the repair was tested by asking the patient to the end of the procedure.

There was no restriction on the type of anesthesia; local, spinal or general anesthesia was employed depending on the surgeon, patient and anesthetic factors. Nursing staff recorded pain scores in first 24 hours postoperatively. Analgesic use was also recorded. Patients were then seen as outpatient at 1, 6 weeks and 6 months after operation by training medical staff to check wound healing and any early complications.

\section{Results:}

Majority of the patients $\mathrm{n}=180(56 \%)$ were having indirect inguinal hernia, next was direct $\mathrm{n}=152(30.4 \%)$ and hernia was seen in 68 cases. $n=68(13.6 \%)$. All were hospitalized postoperatively. There was no difference in the various anesthesia groups, the only difference was cost.

Postoperative pain was less and some form of analgesia was needed in $78 \%$ of the patients. There was early return to normal life routine, mean time was 14 days. 196 patients were discharged the following day, 3 went into urinary retention requiring catheterization. 1 patient had a large scrotal hematoma which required re- 
exploration. 2 patients had transient testicular swelling, two had seroma and 2 had stitch sinus. Chronic pain in groin was noticed in 1 patient. Mean operating time was 45 min.

Follow up was made it one week, six weeks and 6 months interval. Only four patients had recurrence, which is $1 \%$ comparable to any study worldwide. The cause of one recurrence was postoperative cough and straining while other three were due to inadequate tightening of the deep ring at the time of re-repair.

\section{Discussion:}

Results of this trial to date indicate that polypropylene mesh has no added advantage over polypropylene darn with respect to early post operative pain, complications or return to normal activities. Furthermore early recurrence was less than mesh repair ${ }^{8}$. This trial does not support the previous studies of mesh repair that suggest open mesh repair has less post operative pain, and results in a quicker return to normal activity ${ }^{9}$. That mesh repairs are associated fewer post operative complications and a lower recurrence rate has not been supported by this study. As mesh is more expensive and, if it becomes infected, may require removal. The results of this study questions is widespread application. The length of time to full recovery in this trail was on average four weeks and was comparable to hernia, repaired with mesh.

In a randomized trial to Shouldice repair compared with darn, recurrence rates were similar; $4 \%$ for the Shouldice method and $1.8 \%$ for the darn ${ }^{10}$.

Moreover the recurrence rate for the Shouldice method was higher in the general surgical practice than the $1.1 \%$ reported from the Shouldice clinic ${ }^{11}$. Long term follow up is required for full determination of recurrence rate. It has been suggested that a minimum of 10 years is needed as $20 \%$ recurrences will not be apparent for 15 years $^{12}$.
It is difficult to organize a trial over that length of time. Early recurrence is likely to represent technical failure i.e. repair and posterior wall and narrowing of deep ring. Other causes include obesity, persistent straining for chronic constipation, urinary retention, coughing, premature resumption of heavy physical work. The recurrence rate in present study is low $1 \%$ at six months but could be higher at one year.

We found out that the best method of primary inguinal hernia repair is polypropylene darn as this is cheap with less post operative pain, can be easily done under any anesthesia, has fewer complications and has acceptable recurrence rate.

\section{References:}

1. George H Sakorafes et al, Open tension free repair of inguinal hernias; Licthenstein technique. BMC surgery 2001;1:3.

2. Perviz K. Amid, Medscape Surgery, 2001; 3(2).

3. Raphael Rosch et al. BMC Medical Genetics 2002; 3-2.

4. Bendavid R. New techniques in hernia repair. World J Surgery $1989 ; 13-522$.

5. Maloney GE, Gill WG, Barclay RC. Operation for hernia: technique of nylon darn. Lancet 1948; 2-45.

6. Maloney GE. Results of nylon darn repair of Herniae. Lancet 1958; 1-273.

7. Lichtension IL, Shulman AG, Amid PK, Montllor M. The tension free hernioplasty. Am J Surg 1989; 157-188.

8. Koukourou W, Lyon J. Rice and DA Wattchow. Prospective randomized trial of polypropylene mesh compared with nylon darn in inguinal hernia repair. Br J Surg 2001; 88-931.

9. Amid PK, Shulman AG, Licthtenstein IL. Critical scrutiny of the open tension. Free hernioplasty. Am J Surg 1993; 165-369.

10. Kingsworth AN. Gray MR. Nott DM. Prospective randomized trail comparing the Shouldice technique and plication darn for inguinal hernia. Br J Surg 1992; 79-1068.

11. Glassow F. The Shouldice hospital technique. Int Surg 1986. 71-148.

12. Ravitch MM, Repair of hernias. Chicago, Illinosis: Year Book Medica 1969. 